\title{
Estimation of primaries and near-offset reconstruction by sparse inversion: Marine data applications
}

\author{
G. J. A. van Groenestijn ${ }^{1}$ and D. J. Verschuur ${ }^{1}$
}

\begin{abstract}
Most wave-equation-based multiple removal algorithms are based on prediction and subtraction of multiples. Especially for shallow water, the prediction strongly relies on a correct interpolation of the missing near offsets. The subtraction of predicted multiples from the data can easily lead to the distortion of primaries if primaries and multiples overlap. Recently, a new approach for surface-related multiple removal was proposed: the estimation of primaries by sparse inversion (EPSI), which is based on a full waveform inversion approach. EPSI is based on the same primary-multiple model as surface-related multiple elimination (SRME) and does not require a subsurface model. In contrast to SRME, EPSI estimates the primaries as unknowns in a multidimensional inversion process rather than a subtraction process.
\end{abstract}

The multidimensional primary impulse responses are parameterized by band-limited spikes, which are estimated such that they, along with their corresponding multiples, match the input data. An interesting aspect of the EPSI method is that it produces a residual, which is the part of the input data not explained by primaries and multiples. This residual can be analyzed and may provide useful information on the primary estimation process. Furthermore, it has been demonstrated that EPSI is also capable of reconstructing the missing near offsets from the multiples. The proposed method is applied to a field data set with moderate water depth, where it is demonstrated that the results are comparable with SRME. This data set is used to illustrate the residual. For a shallow-water field data set, it is shown that EPSI gives a better result than the standard SRME result caused by EPSI's capability to reconstruct the missing near offsets.

\section{INTRODUCTION}

Surface-related multiples still pose an important problem on marine seismic data. Over the last two decades, several approaches for wave-equation-based multiple prediction and subtraction have been proposed: model-based methods (Berryhill and Kim, 1986; Wiggins, 1988; Lokshtanov, 1999) and data-driven methods, such as surface-related multiple elimination (SRME) (Verschuur et al., 1992; Berkhout and Verschuur, 1997; Weglein et al., 1997). Both approaches involve an adaptive subtraction process in which unknowns in the method (water-bottom reflectivity or the source wavelet) are estimated. The subtraction process is not obvious, as primaries can easily be distorted if multiples and primaries overlap (Guitton and Verschuur, 2004).

In van Groenestijn and Verschuur (2009a), we have introduced the estimation of primaries by sparse inversion (EPSI) and its application to near-offset reconstruction. The main difference with the traditional prediction and subtraction approaches is that EPSI con- siders the primary impulse responses as the unknowns in a largescale inversion process. The primary impulse responses are parameterized by band-limited spikes. They are estimated in such a way that they, along with their corresponding multiples, explain the input data (i.e., primaries and multiples). The residual, the difference between input data and estimated primaries plus multiples, is driven to zero during the optimization process.

Recasting multiple removal as a large-scale inversion method, in which the primaries are unknowns, is not new. Van Borselen et al. (1996) describe a method that estimates primaries as unknowns in an inversion process, provided that the input wavelet is known. Amundsen (2001) estimates primaries after up/down decomposition by a multidimensional division of the upgoing by the downgoing wavefields, provided that the direct wave, especially at the near offsets, is measured in the downgoing waves. EPSI operates on data where the direct wave is removed and does not need to know the source wavelet because it will estimate it. Other inversion methods that operate on data in which the direct wave is removed and do not

Manuscript received by the Editor 27 January 2009; revised manuscript received 29 April 2009; published online 11 December 2009.

${ }^{1}$ Delft University of Technology, The Netherlands. E-mail: G.J.A.vanGroenestijn@tudelft.nl; D.J.Verschuur@tudelft.nl. (C) 2009 Society of Exploration Geophysicists. All rights reserved. 
need to know the source wavelet are described by Biersteker (2001) and by Berkhout (2006). The 2D-Decon method as described by Biersteker (2001) estimates the (missing) shallow primaries under a minimum energy constraint of the final primary output. Berkhout (2006) obtains the inverse primaries and the inverse source wavelet by separating them in the inverse data space, which requires a multidimensional inversion. In contrast to 2D-Decon, EPSI estimates all the primaries simultaneously, not just in the shallow part, and it does not assume that the primaries have minimum energy. Finally, unlike the inverse data-space method (Berkhout, 2006), EPSI does not require a transformation to the inverse data space.

In each iteration, EPSI tries to explain more of the data in terms of primaries and their corresponding multiples. It is possible to continue to iterate until the residual between the input data and the predicted data is zero. However, it may be interesting to stop before that point is reached to leave unexplained energy, e.g., resulting from 3D effects or amplitude inconsistencies, in the residual. This is in contrast to conventional multiple removal methods, which can only divide the data into primaries and multiples.

Especially in shallow-water situations, the wave-equation-based prediction suffers from the fact that the near offsets are not measured and thus need to be interpolated before the multiple prediction process is applied. This means that wrongly interpolated near offsets will produce errors in the predicted multiples and therefore limit the quality of the primary output. As demonstrated by van Groenestijn and Verschuur (2009a), EPSI can use the multiples to reconstruct the missing near offsets. Therefore, EPSI performs well on estimating primaries on shallow-water data. Another data-driven reconstruction method is the pseudoprimary method (Shan and Guitton, 2004), in which a multidimensional autocorrelation of the data is used to fill the near-offset gap. Curry and Shan (2008) improved the pseudoprimary method by extending it with prediction-error filters. However, this improvement does not exclude crosscorrelation artifacts from the missing near offsets completely.

After reviewing the EPSI method, we will discuss the role of the residual when we apply EPSI to a marine data set with moderate water depth. Next we will review the modified EPSI method that can reconstruct missing near-offset data simultaneously while estimating the primaries. This algorithm is applied to a shallow-water marine

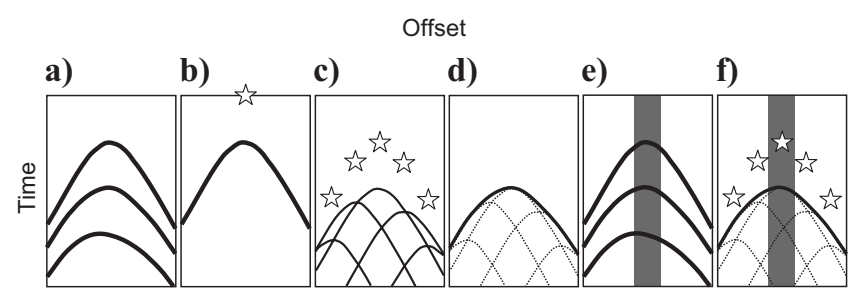

Figure 1. A schematic illustration of the relations between primaries and multiples. (a) A shot gather taken from a data set with one single reflector. (b) The primary event in the shot gather is the consequence of firing the source. (c) The upgoing data will reflect at the surface and generate the multiples. The same multiples are obtained when in each receiver location a secondary source is present, which is fired at the time the primary event reaches the receiver. These secondary sources of the primary event are depicted as stars. (d) The multiples are the result of adding all the delayed primaries. (e) The same shot gather as in (a). The shaded area indicates the offset gap in the data. (f) The first-order multiple is built from delayed primaries caused by secondary sources. The secondary source inside the missing data gap has not been measured, but its consequences have an effect outside the gap. data set. The results are compared with iterative SRME applied to the same data set with interpolated near offsets.

\section{THE PRIMARY-MULTIPLE MODEL AND ITERATIVE SRME}

In the detail-hiding operator notation for 2D data (Berkhout, 1982), a bold quantity represents a prestack data volume for one frequency; columns represent monochromatic shot records, and rows represent monochromatic common receiver gathers. With the use of this notation, we can express the upgoing data, $\mathbf{P}$, as

$$
\mathbf{P}=\mathbf{X}_{0} \mathbf{S}+\mathbf{X}_{0} \mathbf{R P}
$$

where $\mathbf{X}_{0}$ is an impulse response from a dipole pressure source to monopole receivers. The matrix $\mathbf{X}_{0}$ multiplied by the source properties matrix, $\mathbf{S}$, equals the primaries

$$
\mathbf{P}_{0}=\mathbf{X}_{0} \mathbf{S}
$$

Note that what are called "primaries" in this paper actually refer to all events that have not reflected at the surface, meaning that internal multiples and converted waves are also included. The matrix multiplication of $\mathbf{X}_{0}$ by the reflection at the surface, $\mathbf{R}$, and the data $\mathbf{P}$ will result in the surface multiples

$$
\mathbf{M}=\mathbf{X}_{0} \mathbf{R P} .
$$

Figure 1a-d illustrates this forward model of primaries and multiples in terms of secondary or Huygens' sources (see also Verschuur, 1992).

From equation 1, it can be derived that surface multiples can be predicted by a multidimensional convolution of the primaries with the total data and with the surface operator, $\mathbf{A}$,

$$
\mathbf{M}=\mathbf{P}_{0} \mathbf{A P},
$$

where $\mathbf{A}=\mathbf{S}^{-1} \mathbf{R}$. Because the primaries are not known beforehand, the relationship is written as an iteration process (Berkhout and Verschuur, 1997):

$$
\hat{\mathbf{P}}_{0, i+1}=\mathbf{P}-\hat{A}_{i+1} \hat{\mathbf{P}}_{0, i} \mathbf{P},
$$

where $i$ represents the iteration number, $\mathbf{A}$ is replaced by $A(\omega) \mathbf{I}$ and $\hat{\mathbf{P}}_{0,1}=\mathbf{P}$. To avoid the use of an obliquity factor (Weglein et al., 1997) no deghosting is done on the data for the sources (Verschuur, 1991). Because there are more unknowns, $\hat{\mathbf{P}}_{0, i+1}$ and $\hat{A}_{i+1}$, than knowns, $\mathbf{P}$, in equation 5 , an extra constraint is needed. Typically, it is assumed that the primaries have minimum energy (the L2 norm). This constraint is used when $\hat{A}_{i+1}$ is estimated as a filter that matches the predicted multiples, $\hat{\mathbf{P}}_{0, i} \mathbf{P}$, to the data in the time domain, resulting in the new primary estimation, $\hat{\mathbf{P}}_{0, i+1}$ (Verschuur and Berkhout, 1997). The minimum-energy norm often leads to the correct subtraction but does not work properly in all cases (Nekut and Verschuur, 1998). Guitton and Verschuur (2004) and van Groenestijn and Verschuur (2008) have shown that other minimization norms, such as the L1 norm, or the Cauchy norm can lead to different and sometimes better subtraction results. 


\section{ESTIMATION OF PRIMARIES BY SPARSE INVERSION}

The proposed EPSI method, described by van Groenestijn and Verschuur (2009a), estimates primaries through sparse inversion, and therefore, it does not involve an adaptive subtraction of the predicted multiples. In fact, primaries and their corresponding multiples are predicted such that they explain the total data.

The various steps in the EPSI algorithm are illustrated with a field data set with moderate water depth. Figure 2a shows one shot gather selected from the 2D line. Note that those data are acquired in an area with a bottom with moderate water depth. In Appendix A, the preprocessing steps applied to these data are described. Furthermore, some implementation details of the SRME and EPSI algorithms can be found there.

The algorithm is based on the same primary-multiple model, as discussed above. If we take $\mathbf{S}=S(\omega) \mathbf{I}$ (meaning a constant source wavelet for all shots and no source directivity effects) and $\mathbf{R}=-\mathbf{I}$, equation 1 becomes

$$
\mathbf{P}=\mathbf{X}_{0} S-\mathbf{X}_{0} \mathbf{P}
$$

This equation has more unknowns, $\mathbf{X}_{0}$ and $S$, than knowns, $\mathbf{P}$, and therefore, an extra constraint is needed to solve it. We use the constraint that $\mathbf{X}_{0}$ is sparse in the time domain. We assume that $\mathbf{X}_{0}$ can be represented by a limited number of spikes with large amplitudes (from the major reflecting boundaries) and many small amplitude spikes (from all other events).

To ensure that the multiples have enough energy to play a role in this process, a gain $e^{\gamma t}(t$ is the time and $\gamma$ is positive constant) is applied to each trace. Note that with such an exponential gain, the convolutional relationship between primaries and multiples remains intact. The objective function $J$ is introduced as

$$
J_{i}=\sum_{\omega} \sum_{j, k}\left|\mathbf{P}-\hat{\mathbf{X}}_{0, i} \hat{S}_{i}+\hat{\mathbf{X}}_{0, i} \mathbf{P}\right|_{j, k}^{2}
$$

where $i$ denotes the iteration number, $\Sigma_{j, k}$ indicates a summation over all the squared elements of the matrix (i.e., a summation over all sources and receivers), and $\Sigma_{\omega}$ indicates a summation over all the frequencies. Note that the objective function will go to zero if the correct $\hat{\mathbf{X}}_{0}$ and $\hat{S}$ are found. This is in contrast to SRME, in which usually minimum energy of the primaries is assumed. The objective function is minimized iteratively by estimating $\hat{\mathbf{X}}_{0}$ and $\hat{S}$ in alternate steps. The initial values of $\hat{\mathbf{X}}_{0}$ and $\hat{S}$ are set to zero. The update, $\Delta \mathbf{X}_{0}$, is a steepest descent step, obtained by taking the derivative of $J$ with respect to the elements of $\hat{\mathbf{X}}_{0}$ :

$$
\Delta \mathbf{X}_{0}=\left(\mathbf{P}-\hat{\mathbf{X}}_{0, i} \hat{S}_{i}+\hat{\mathbf{X}}_{0, i} \mathbf{P}\right)\left(\hat{S}_{i} \mathbf{I}-\mathbf{P}\right)^{H},
$$

where $\left(\hat{S}_{i} \mathbf{I}-\mathbf{P}\right)^{H}$ is the complex adjoint of $\left(\hat{S}_{i} \mathbf{I}\right.$ $-\mathbf{P})$. The term $\left(\mathbf{P}-\hat{\mathbf{X}}_{0, i} \hat{S}_{i}+\hat{\mathbf{X}}_{0, i} \mathbf{P}\right)$ represents the unexplained data or the residual. The matrix

$$
\hat{\mathbf{X}}_{0, i+1}=\hat{\mathbf{X}}_{0, i}+\alpha \Delta \overline{\mathbf{X}}_{0}
$$

multiplication $\left(\mathbf{P}-\hat{\mathbf{X}}_{0, i} \hat{S}_{i}+\hat{\mathbf{X}}_{0, i} \mathbf{P}\right)\left(\hat{S}_{i} \mathbf{I}\right)^{H}$ can be interpreted as information of primaries in the residual that has not yet been explained by the algorithm. The matrix multiplication $\left(\mathbf{P}-\hat{\mathbf{X}}_{0, i} \hat{S}_{i}+\hat{\mathbf{X}}_{0, i} \mathbf{P}\right)($ $-\mathbf{P})^{H}$ can be interpreted as multiples in the residual that are mapped to the primary locations. Thus, both multiples and primaries are simultaneously used to update the primary impulse responses.

In EPSI, the sparseness is imposed on the update of $\hat{\mathbf{X}}_{0}$. First the update $\Delta \mathbf{X}_{0}$ is calculated according to equation 8. Next, $\Delta \mathbf{X}_{0}$ is divided by $\sqrt{\omega} e^{(i \pi / 4)}$, after which it is transformed to the time domain. Figure $2 \mathrm{~b}$ shows this result for the same shot gather. The term $\sqrt{\omega} e^{(i \pi / 4)}$ represents the frequency-dependent behavior of a two-dimensional dipole source (Berkhout, 1982). By dividing out this term, the events in $\Delta \mathbf{X}_{0}$ will have a zero-phase appearance in the time domain. Note that because both $\hat{\mathbf{X}}_{0}$ and $\hat{S}$ are zero in the first iteration step, $\Delta \mathbf{X}_{0}$ equals a multidimensional correlation of the data with itself, $-\mathbf{P} \mathbf{P}^{H}$. Next, a suitable time window is placed over $\Delta \mathbf{X}_{0}$ in the time domain, and the event(s) per trace with the largest amplitudes are selected. The result of this procedure is displayed in Figure 2c. By increasing the size of the window in each iteration, convergence is improved. In each iteration, the window should exclude strong events in the update not associated with primaries (e.g., the event pointed out by the arrow in Figure $2 b$ ) as much as possible. The primaries precede the multiples that they create. A window will allow us to select mainly primaries. When the window is increased so far that it includes the position of the multiples, then the multiples are largely removed from the residual. The starting size of the window and the speed with which the window increases are therefore a function of the position of the dominant primaries and dominant multiples. The window will also exclude noncausal events $(t<0.25 \mathrm{~s})$.

After bringing $\Delta \mathbf{X}_{0}$ back to the frequency domain, it is multiplied by the factor $\sqrt{\omega} e^{(i \pi / 4)}$ to restore the 2D dipole behavior. The sparse update, $\Delta \overline{\mathbf{X}}_{0}$, is added to the primary impulse response:
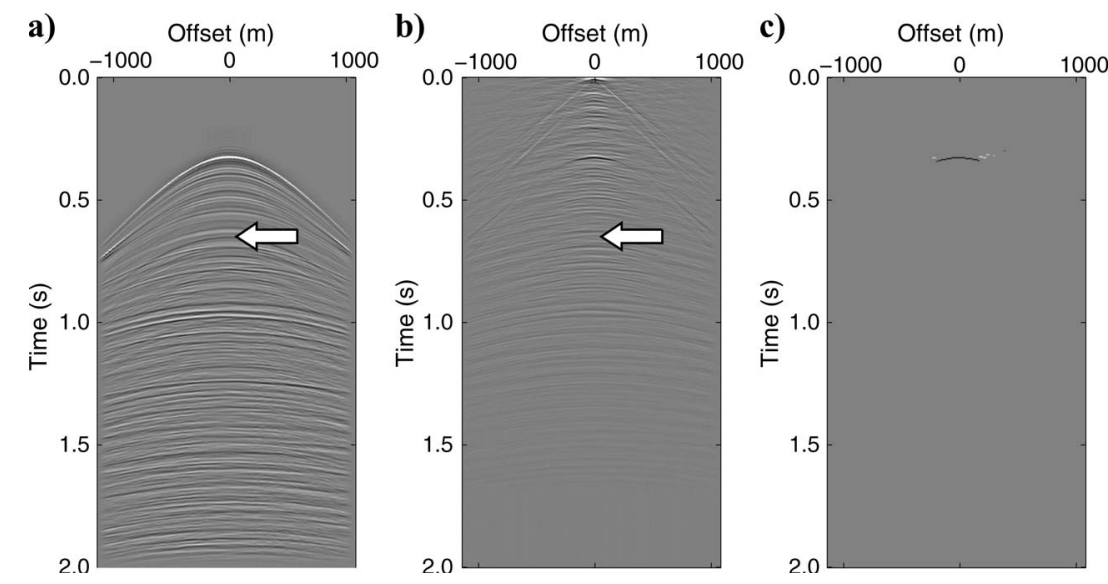

Figure 2. (a) Input shot gather with multiples from the marine data with moderate water depth. (b) The corresponding update of the primary impulse response $\left(\Delta \mathbf{X}_{0}\right)$. (c) The corresponding update of the primary impulse response after windowing and applying sparseness $\left(\Delta \overline{\mathbf{X}}_{0}\right)$. Both arrows indicate the position of the first-order water-bottom multiple at zero offset. 
where $\alpha$ is a positive frequency-independent factor that scales the update step in such a way that the objective function value decreases.For the examples in this paper, we have used the method presented in van Groenestijn and Verschuur (2009a) to determine $\alpha$. A more advanced way to estimate $\alpha$ might be by matching $\alpha \Delta \overline{\mathbf{X}}_{0}\left(S_{i}-\mathbf{P}\right)$ to $\mathbf{P}+\hat{\mathbf{X}}_{0, i}\left(S_{i}-\mathbf{P}\right)$. Throughout the process, the value of $\alpha$ should not vary too much. Large variations in the beginning of the process are a sign of multiples being too weak compared to the primaries. Large variations at the end may be an indication that the iteration process is better stopped.

The update of $\hat{S}$ is executed in the same way as the update of $\hat{\mathbf{X}}_{0}$ :

$$
\Delta \mathbf{S}=\hat{\mathbf{X}}_{0, i+1}^{H}\left(\mathbf{P}-\hat{\mathbf{X}}_{0, i+1} \hat{S}_{i}+\hat{\mathbf{X}}_{0, i+1} \mathbf{P}\right)
$$

From the full matrix $\Delta \mathbf{S}$, the diagonal elements are selected and summed to get the scalar $\Delta S . \Delta S$ is brought to the time domain, where its length is limited. After that, the update is scaled to ensure that the next objective function of equation 7 is lower than the previ-

a)
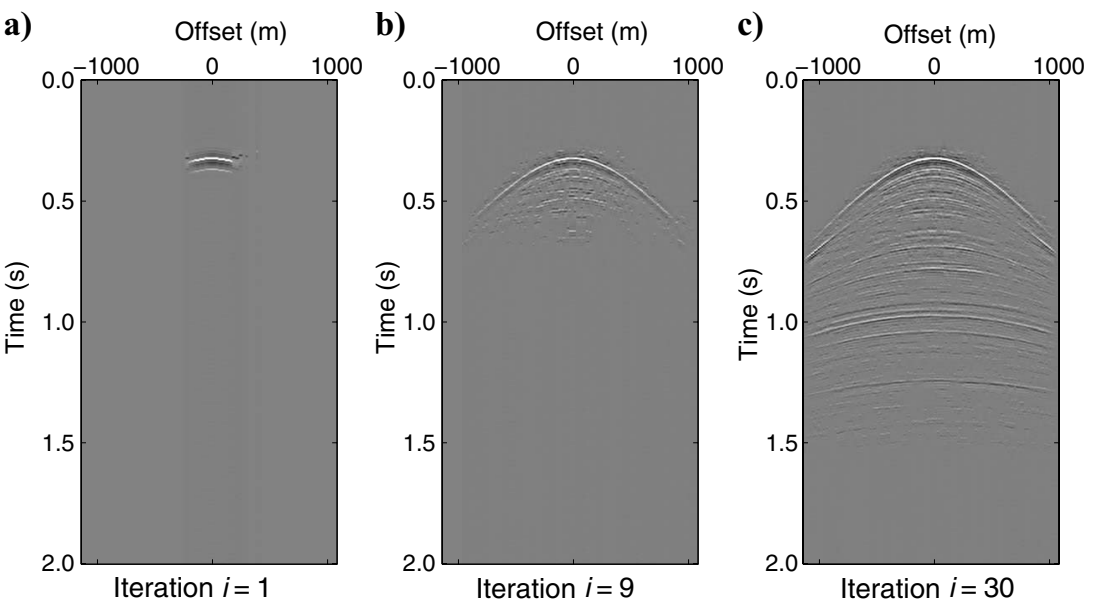

Figure 3 . The primary estimation, $\hat{\mathbf{X}}_{0, i} \hat{S}_{i}$, during different iterations. a)

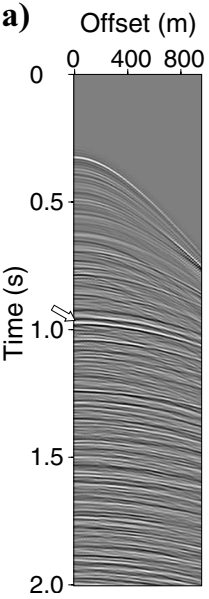

b)

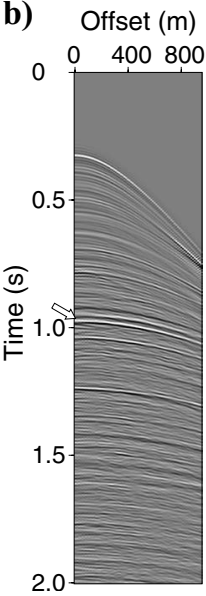

c)

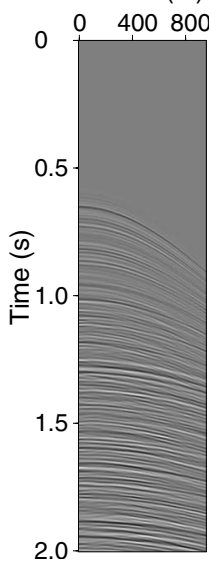

d)

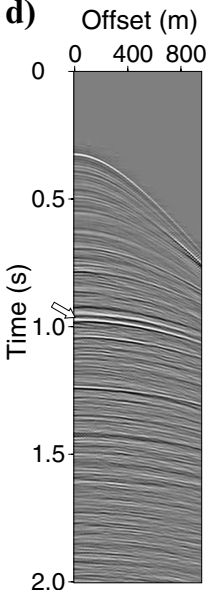

ous one. Figure 3 shows the progression of the primary estimation during various iterations.

\section{RESULTS ON MARINE DATA WITH MODERATE WATER DEPTH}

The estimates of $\hat{\mathbf{X}}_{0}$ and $\hat{S}$ can be used in two ways to come to a primary estimation: a direct way, by convolving the estimated impulse responses with the estimated wavelets, $\hat{\mathbf{P}}_{0}=\hat{\mathbf{X}}_{0} \hat{S}$, and a conservative way, by creating the multiples and subtracting them from the data, $\hat{\mathbf{P}}_{0}=\mathbf{P}-\hat{\mathbf{M}}=\mathbf{P}+\hat{\mathbf{X}}_{0} \mathbf{P}$. Note that the estimated multiples are simply subtracted from the data without any matching filter. The difference between the two options is that in the second option, the residual data are added to the direct primary estimate. The direct primary estimation for the shot gather under consideration (Figure 4a) is displayed in Figure 4d. The corresponding estimated multiples are plotted in Figure 4e. Iterative SRME has been applied to the same data set (Figure $4 \mathrm{~b}$ and c). Both SRME and EPSI have removed the waterbottom multiple. It is interesting to look at the strong reflection at about $1.0 \mathrm{~s}$ in the primaries at the near offsets (indicated by arrows). One can observe that SRME has weakened this primary.

The stacks in Figure 5 compare the direct primary estimate of EPSI with the SRME result. Figure 6 zooms in on the region between 2.2 and $3.0 \mathrm{~s}$ and shows that there is a diagonal multiple residual in the SRME output at about $2.4 \mathrm{~s}$ (top arrow). Another multiple is visible at about $2.7 \mathrm{~s}$ on the right side of the stack (bottom arrow). These multiples are not very strongly present in the EPSI output, although none of the events in the EPSI output in Figure $6 \mathrm{~b}$ is very strong. This is especially true for the flat event at $2.6 \mathrm{~s}$ (middle arrow).

The stacked residual in Figure $7 \mathrm{c}$ shows the events in the data that are not yet explained by the model. A good example is the strong reflector just above $1.0 \mathrm{~s}$ (top arrow). Note also the residual dipping multiples at about $3 \mathrm{~s}$ (middle arrow) and the low frequencies below $3.5 \mathrm{~s}$ (bottom arrow). If we continue to iterate, this residual will go to These fake primaries will cause multiples at later times. These fake multiples can then be compensated for by explaining them as primaries. By continuing the iterations, the errors are pushed out of the time window.

An answer to this unwanted effect can be to stop iterating before the residual is zero. Maybe equation 9 can play a role here. A sparse update, $\Delta \overline{\mathbf{X}}_{0}$, without noise will have a bigger impact on the objective function when added to the primary impulse responses (it is explaining primaries and multiples) than the impact of a sparse update with only noise on the objective function (it is explaining noise in the primaries and is creating noise in the multiples). Therefore, a sparse update without noise will have a bigger scaling factor, $\alpha$, than a sparse update with noise. A decrease in the scalzero. Noise in the data is explained as primaries.
Figure 4. (a) A shot gather of the moderately deepwater marine data set with multiples. (b) (d) The direct primary estimate obtained with the EPSI method. (e) The estimated multiples from the EPSI method. e) Offset $(\mathrm{m})$

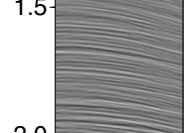

2.0 
ing factor at the end of the process might therefore be a sign that the method is trying to explain noise. Another answer may be to disallow the creation of primaries in a certain area, for example below $3 \mathrm{~s}$. The correct primary estimation will then leave a residual that would consist of the primaries below $3 \mathrm{~s}$. In fact, the EPSI process can be used as a quality control tool to check the consistency of the input data. If the residual goes to zero smoothly, the data are all right. If there are clear events residing in the residual data, these may be an indication that something is not consistent in the data, which may be resolved by changing the preprocessing sequence. This is a topic for further investigation.

\section{RECONSTRUCTION OF MISSING NEAR OFFSETS}

In this section, we will review the reconstruction of missing near offsets in EPSI, as proposed by van Groenestijn and Verschuur (2009a). The basic EPSI method is extended to the situation ofmissing near offsets. Equation 1 shows how data are built from sources, data (which can be seen as secondary sources), and primary impulse responses. Although the missing near-offset data are not measured, the consequences of "firing" the secondary sources in the near-offset region are measured in the multiples. Therefore, the near-offset data a)

CMP coordinate $(\mathrm{km})$

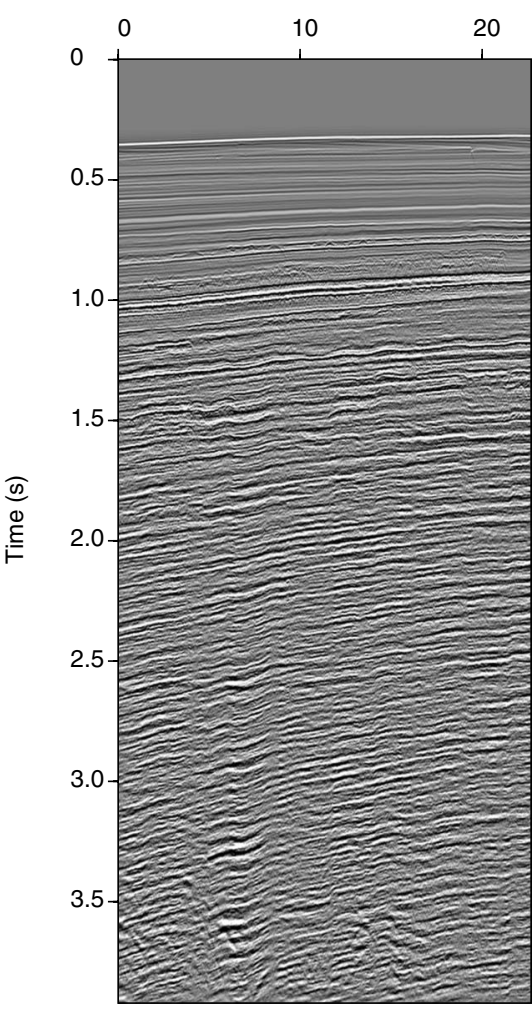

b)

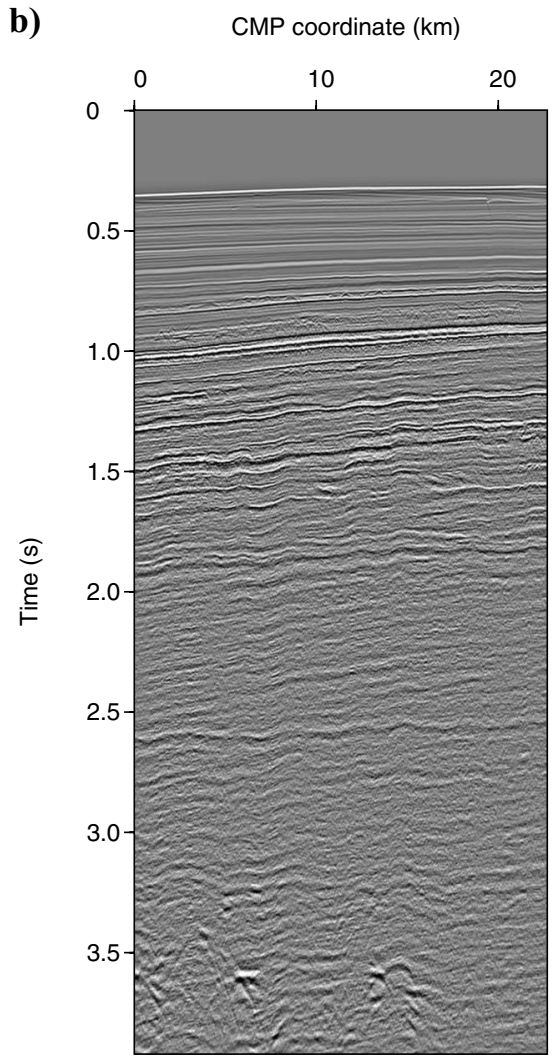

c) CMP coordinate $(\mathrm{km})$

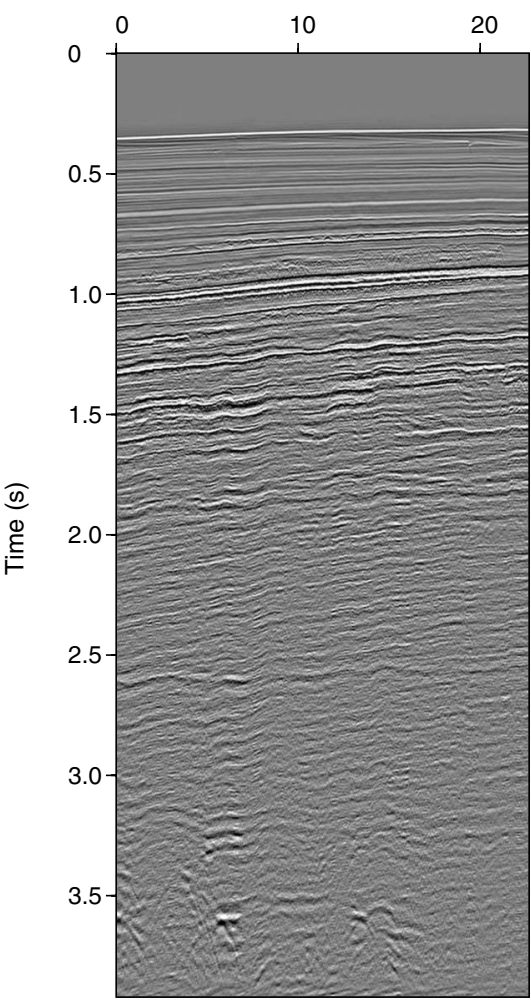

Figure 5. (a) Stacked section of the moderately deepwater marine data with multiples. (b) Stack of the primaries obtained with iterative SRME. (c) Stack of the direct primary estimate from the EPSI method.

a)

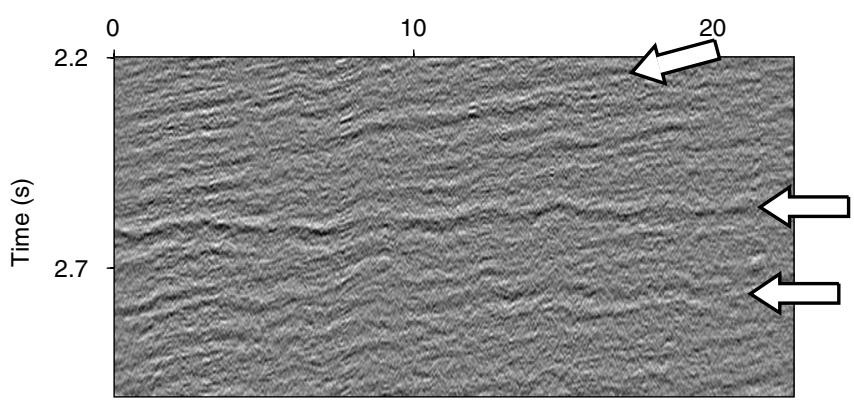

b)

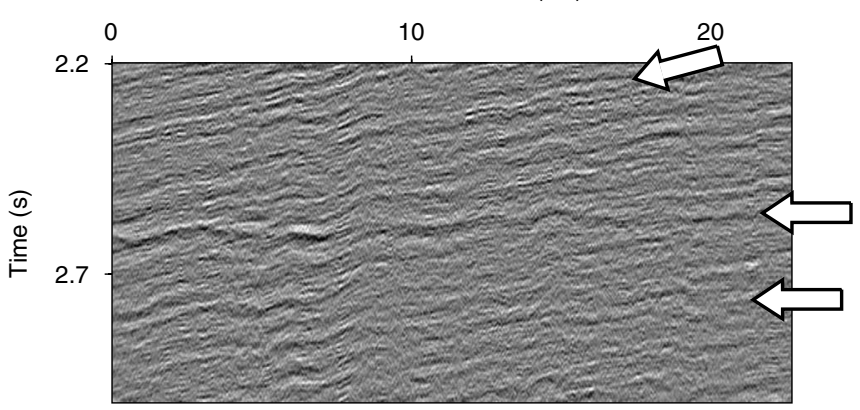

Figure 6. (a) Enlargement of the stack of the SRME primaries (Figure 5b). (b) Enlargement of the stack of the EPSI primaries (Figure 5c). 
can be reconstructed from the multiples. Figure 1e and $\mathrm{f}$ illustrates this. We will discuss the points in the EPSI algorithm for data with missing near offsets that differ from the algorithm for the data without missing near offsets.

The total data obtained in iteration $i$ consists of two subsets: $\mathbf{P}_{i}$ $=\hat{\mathbf{P}}_{i}^{\prime}+\mathbf{P}^{\prime \prime}$, where $\mathbf{P}^{\prime \prime}$ are the data that do not need to be reconstructed, and $\hat{\mathbf{P}}_{i}^{\prime}$ are the missing near-offset data that have to be reconstructed. In the objective function $\hat{\mathbf{P}}^{\prime}, \hat{\mathbf{X}}_{0}$, and $\hat{S}$ are the unknowns:

$$
J_{i}=\sum_{\omega} \sum_{j, k}\left|\mathbf{P}_{i}-\hat{\mathbf{X}}_{0, i} \hat{S}_{i}+\hat{\mathbf{X}}_{0, i} \mathbf{P}_{i}\right|_{j, k}^{2}
$$

For the first iteration, the values for $\hat{\mathbf{X}}_{0}, \hat{S}$, and $\hat{\mathbf{P}}^{\prime}$ are set to zero.

It is interesting to point out that the difference between the 2D-Decon method described by Biersteker (2001) and EPSI is in essence the term $\hat{\mathbf{X}}_{0} \hat{S}$ in equation 11 that is not present in 2D-Decon. This means that 2D-Decon relies on the minimum energy assumption of the primaries.

The iteration process consists of three consecutive steps. First the update of $\mathbf{X}_{0}$ is calculated. It is the same as in equation 8 except that we use the data $\mathbf{P}_{i}$ (with reconstructed near-offset data from the previous iteration) instead of $\mathbf{P}$ :

$$
\Delta \mathbf{X}_{0}=\left(\mathbf{P}_{i}-\hat{\mathbf{X}}_{0, i} \hat{S}_{i}+\hat{\mathbf{X}}_{0, i} \mathbf{P}_{i}\right)\left(\hat{S}_{i} \mathbf{I}-\mathbf{P}_{i}\right)^{H}
$$

The same substitution, $\mathbf{P}_{i}$ for $\mathbf{P}$, is also the only thing that changes for the update of $S$ :

$$
\Delta \mathbf{S}=\hat{\mathbf{X}}_{0, i+1}^{H}\left(\mathbf{P}_{i}-\hat{\mathbf{X}}_{0, i+1} \hat{S}_{i}+\hat{\mathbf{X}}_{0, i+1} \mathbf{P}_{i}\right)
$$

Finally, the update of $\hat{\mathbf{P}}^{\prime}$ is applied, which is given by the following steepest descent step:

$$
\Delta \hat{\mathbf{P}}^{\prime}=-\left(\mathbf{I}+\hat{\mathbf{X}}_{0, i+1}\right)^{H}\left(\mathbf{P}_{i}-\hat{\mathbf{X}}_{0, i+1} \hat{S}_{i+1}+\hat{\mathbf{X}}_{0, i+1} \mathbf{P}_{i}\right) .
$$

Events that arrive before the primary water-bottom reflection are removed from $\Delta \mathbf{P}^{\prime}$ in the time domain along with parts of the data wedo not want to reconstruct (because they are already in $\mathbf{P}^{\prime \prime}$ ). The update of $\hat{\mathbf{P}}^{\prime}$ is scaled to ensure that the next objective function value of equation 11 is lower than the previous one.

\section{RESULTS ON SHALLOW-WATER MARINE DATA}

We demonstrate the above procedure on a shallow-water field data set. The preprocessing steps can be found in Appendix A. Figure 8 a shows one shot record after this preprocessing sequence. Note that the near offsets have been filled by interpolation except for the first $0.4 \mathrm{~s}$. a)

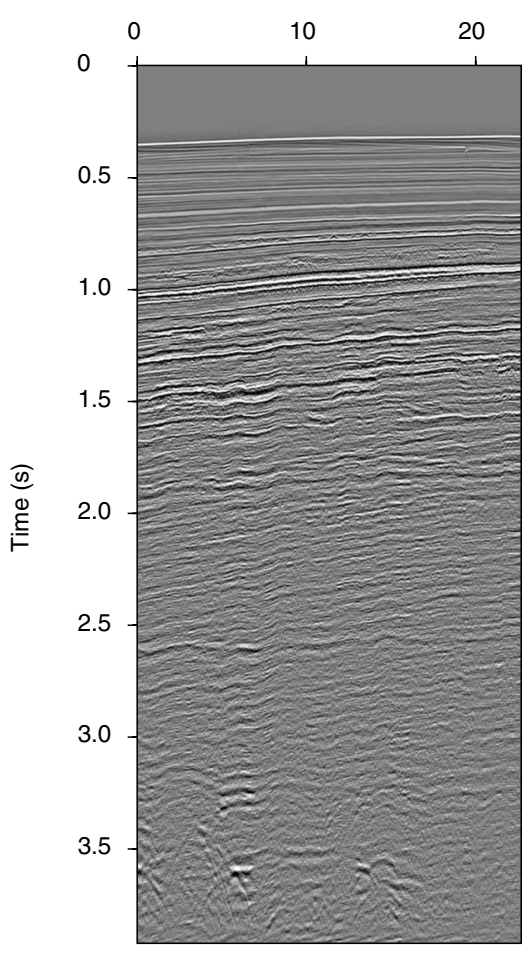

b)

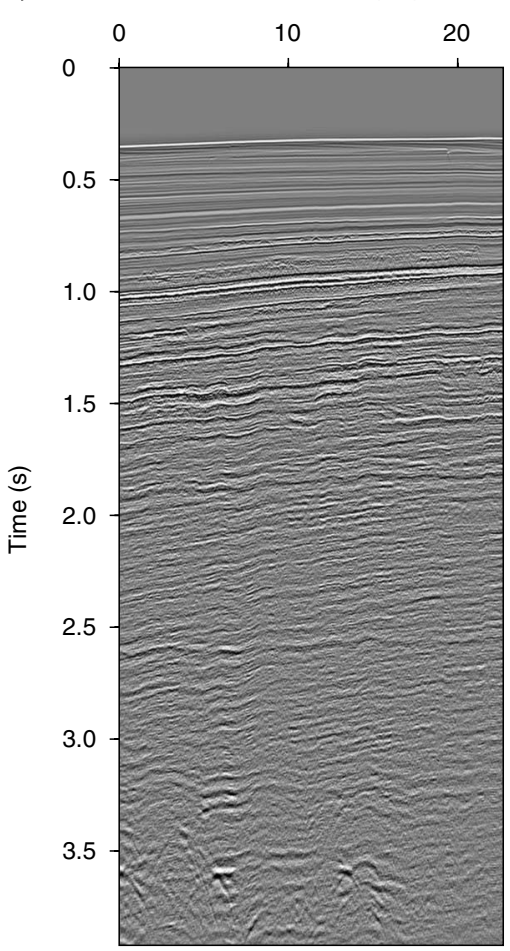

c)
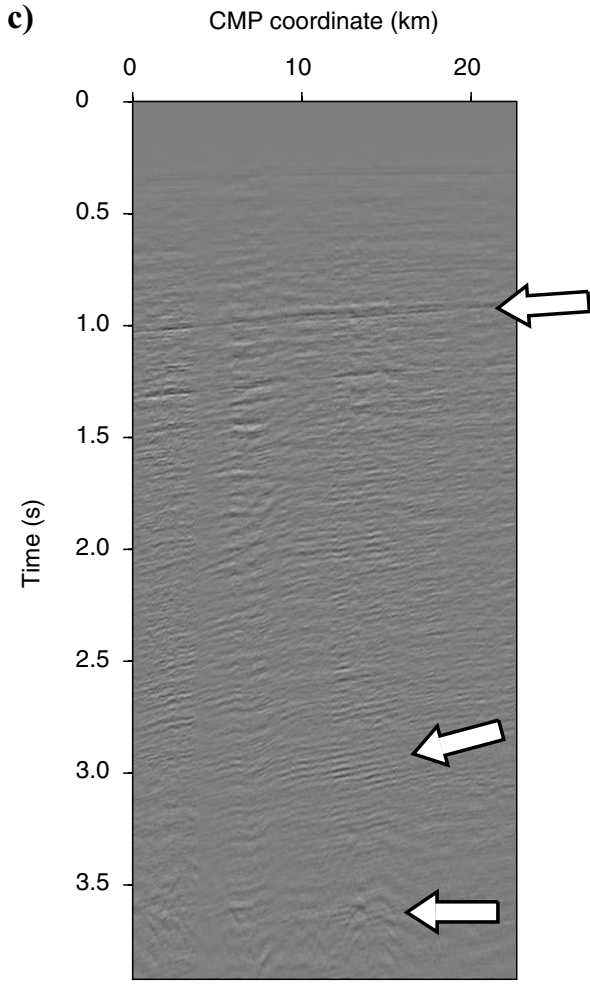

Figure 7. (a) Stacked section of EPSI's direct primary estimate on the marine data with moderate water depth. (b) Stack of the conservative primary estimate of EPSI. (c) Stack of the residual of the EPSI method, i.e., Figure b-a. 
The modified EPSI algorithm, described in the previous section, is applied with $\mathbf{P}^{\prime \prime}$ being the measured data outside the near-offset gap and the interpolated data in the lower part of the missing nearoffset gap (such as shown in Figure 8a). Thus, $\hat{\mathbf{P}}^{\prime}$ is the top part of the near-offset data that are reconstructed. Figure $8 \mathrm{a}-\mathrm{d}$ shows the result of the first iteration of the EPSI algorithm. Note the amount of multiples in Figure 8c that is predicted by a small part of the first reflection only (Figure $8 b$ ). From this result, the importance of having the shallow near-offset data for predicting the multiples becomes clear.
Figure 9 show the final EPSI and SRME results for one shot gather. Because the parabolic Radon interpolation underestimates the strengths of the water-bottom reflection, iterative SRME cannot provide good results. For example, the peg-leg multiple at about $0.7 \mathrm{~s}$ is not properly removed (lower arrows). Note that the wavelet stretch that can be seen in the data (upper arrow) is not present in the EPSI result. Also note that the near offsets in the primary estimation of EPSI are discontinuous in some parts. When these discontinuous parts in the primary impulse responses are convolved with the data, a)

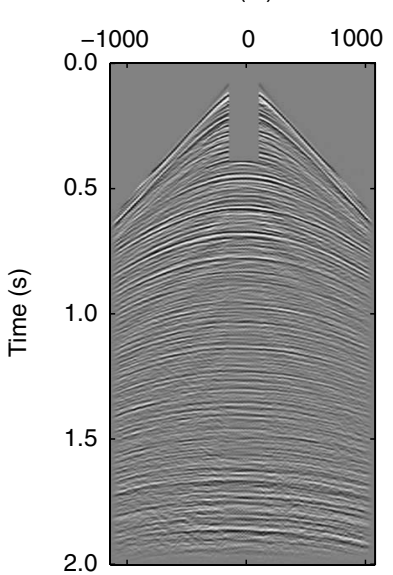

b)

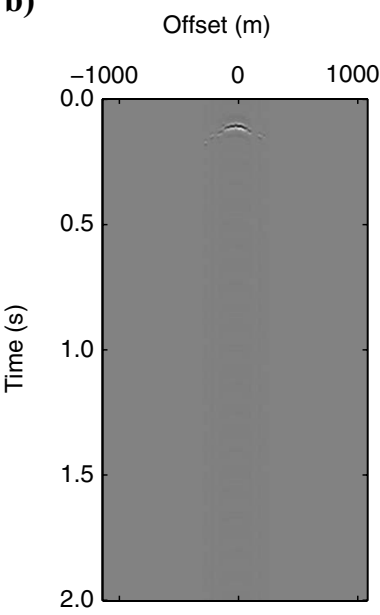

c)

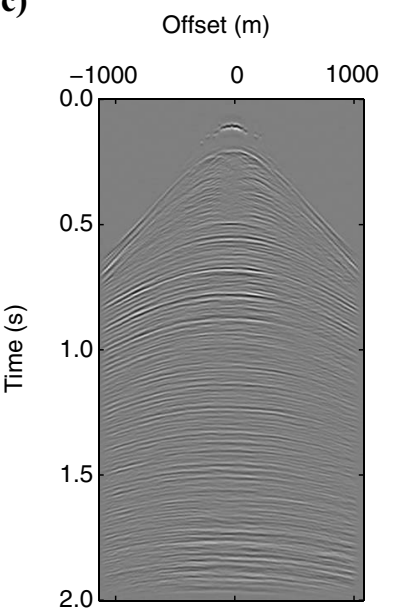

d)

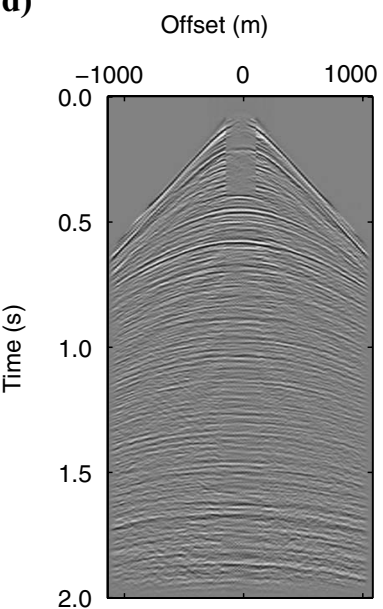

Figure 8. The EPSI result for one iteration. (a) Shot gather with the top part of the near offsets missing. (b) The primary estimate after one iteration. (c) The explained part of the data after one iteration. (d) The residual after one iteration. Note the significant reduction of multiples in (d) solely based on the estimated first primary in (b). This illustrates the importance of a correct near-offset reconstruction.

a)

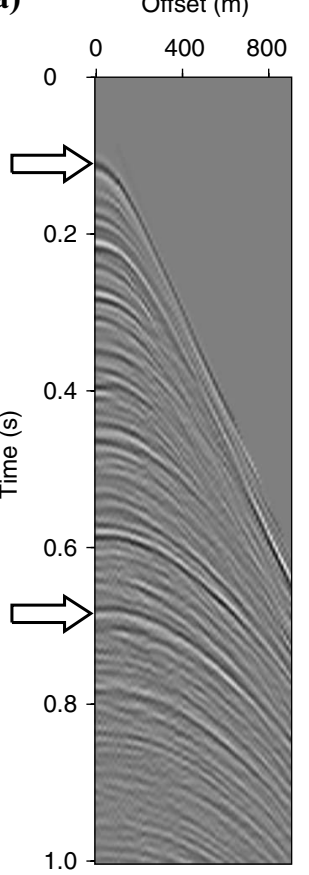

b)

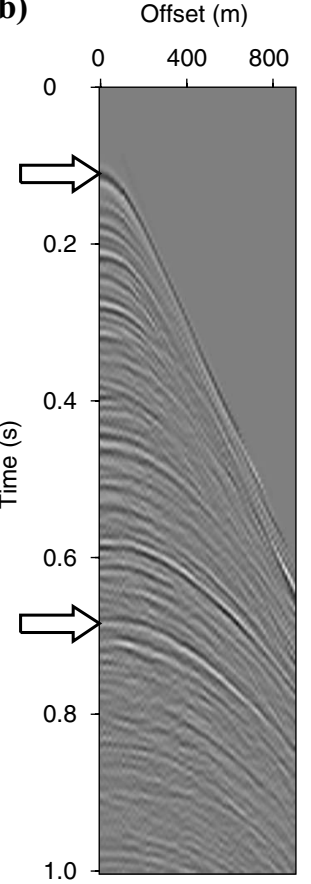

c)

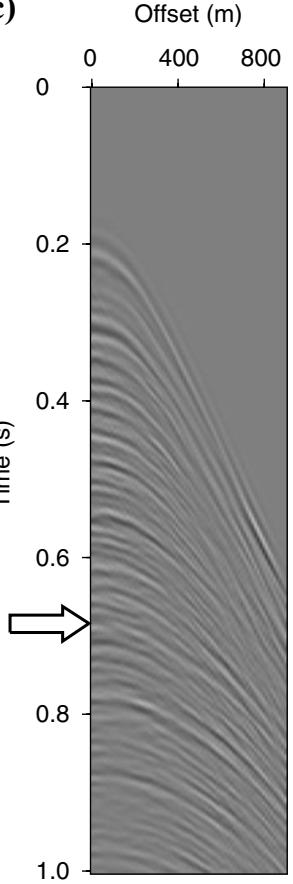

d)

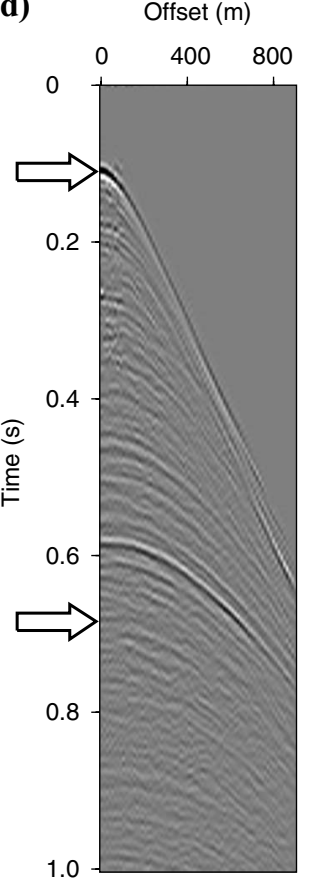

e)
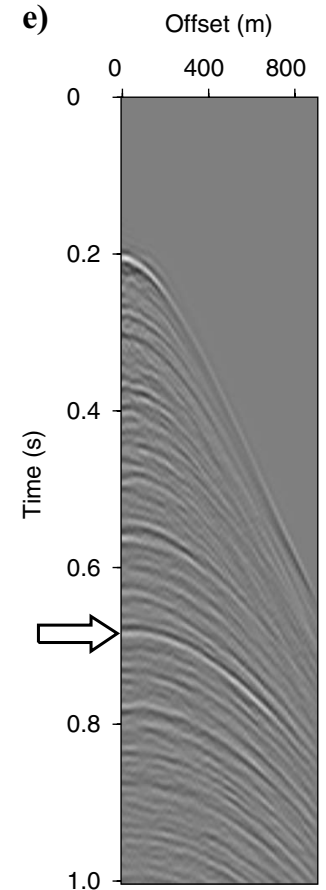

Figure 9. (a) Input shot gather of a shallow-water marine data set with multiples. For the EPSI method, the first $0.4 \mathrm{~s}$ of the interpolated near offsets are set to zero. (b) Primary estimation with iterative SRME. (c) Subtracted multiples of iterative SRME. (d) The direct primary estimate of EPSI. (e) Estimated multiples by EPSI. 
a)

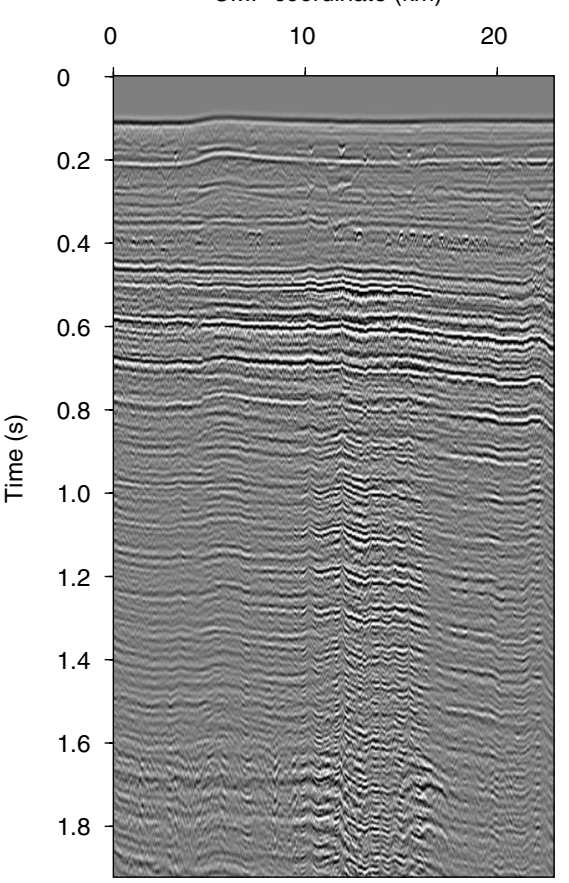

b)

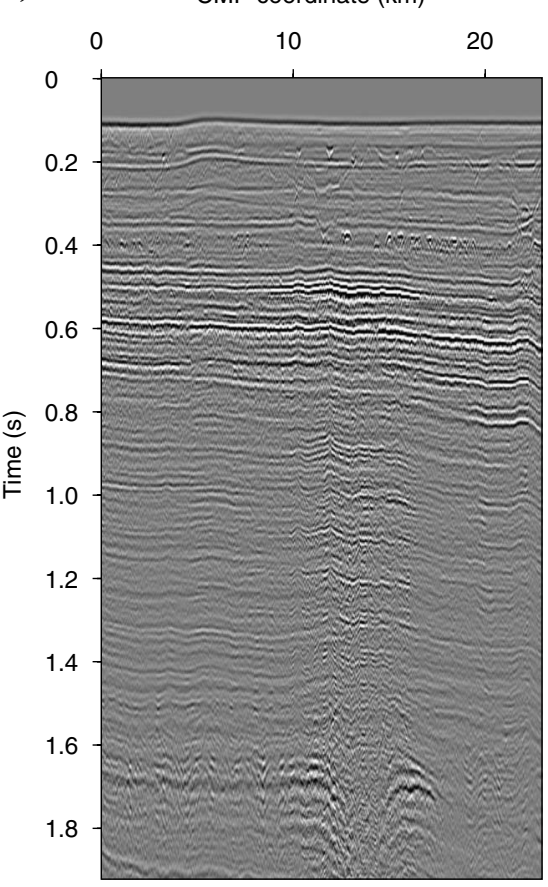

c)

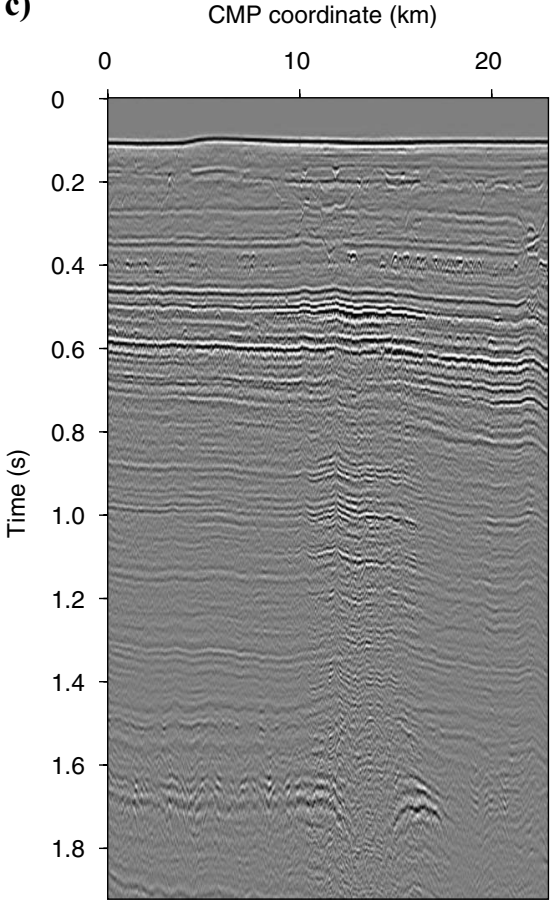

Figure 10. (a) Stacked section of the shallow-water marine data with multiples. (b) The primaries obtained with iterative SRME. (c) The direct primary estimate of EPSI.

a)

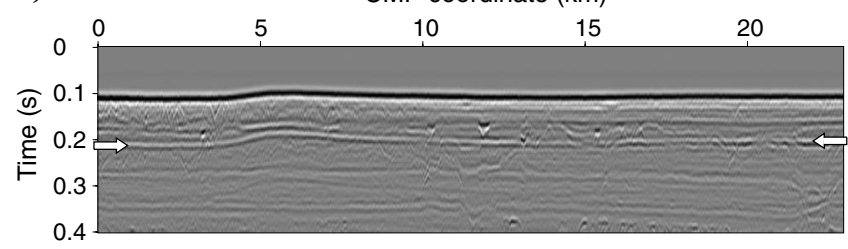

b)

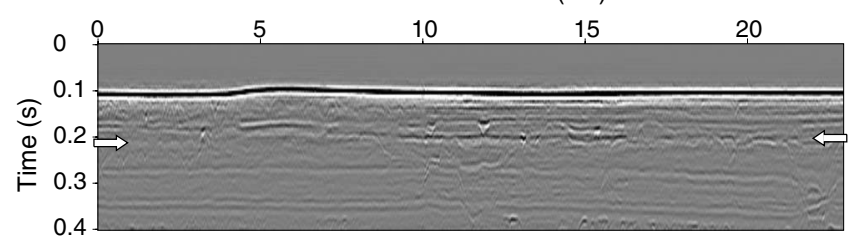

Figure 11.Zooms of the top parts of the stacks of Figure 10. (a) Stack of the SRME primaries. (b) Stack of the EPSI primaries.

they will sum up to create continuous multiples that will match the continuous multiples in the data. So the discontinuous primary impulse responses are a solution of the equations. A smoothing constraint may be used to force the primary impulse responses to be continuous. However, the stack of the primary impulse responses (Figure 10c) shows that a process such as stacking is not hindered by such discontinuous primaries.

The fact that SRME needs correct near-offset data is known (see, e.g., Verschuur, 2006). To bypass the influence of the shallow nearoffset reflections, it is common practice to remove multiples related to shallow primaries with a deconvolution first (Biersteker, 2001) and then apply SRME to those data without the shallow primaries.
However, deconvolution relies on minimum energy, and the deconvolution and SRME processes are two separate steps, although their multiples interfere with each other. This can provide suboptimum results. This is in contrast to EPSI that does not rely on minimum energy in the primaries and estimates all primaries and multiples in one step.

Stacks were made for the complete line, as shown in Figure 10. The estimated near offsets have been included in the stacking process. Except for the shallow events, the result was similar to stacking without the near offsets. This was done to further emphasize the effect of the near offsets as reconstructed by the EPSI method. As can be observed, the water bottom has had a tremendous impact on the multiple-removal results. The area between 1.0 and $1.5 \mathrm{~s}$ is a lot cleaner in the EPSI result (Figure 10c) as compared to the SRME result (Figure 10b). Furthermore, the structure at about $1.7 \mathrm{~s} \mathrm{just} \mathrm{right}$ of the middle is much more visible for the EPSI result. A zoom on the water-bottom area is made in Figure 11. A residual of the first-order water-bottom multiple (between the arrows) is clearly visible in the SRME result (Figure 11a), whereas the EPSI result (Figure 11b) now reveals the reflector that was partly covered by the first-order multiple in the data.

\section{DISCUSSION}

To gain some insights in the convergence of the method, we calculate the effect of varying the final estimated $\hat{S}$ and $\hat{\mathbf{X}}_{0}$ in the objective function

$$
J(\lambda, \mu)=\sum_{\omega} \sum_{j, k}\left|\mathbf{P}-\lambda \mu \hat{\mathbf{X}}_{0} \hat{S}+\lambda \hat{\mathbf{X}}_{0} \mathbf{P}\right|_{j, k}^{2},
$$

where $\lambda$ is a scalar that scales the final estimated $\hat{\mathbf{X}}_{0}$, and $\mu$ is a scalar that scales the final estimated $\hat{S}$. To save computation time, we will 
not calculate equation 15 for the total data $(\mathbf{P})$, direct primaries $\left(\hat{\mathbf{X}}_{0} \hat{S}\right)$, and multiples $\left(\hat{\mathbf{X}}_{0} \mathbf{P}\right)$ but for the stacked data and primaries in Figure 10 after $0.4 \mathrm{~s}$ and stacked multiples. In Figure 12, the effects of varying both $\lambda$ and $\mu$ between 0.1 and 10 can be seen. There appears to be a clear minimum in $\lambda=1$ and $\mu=1$. The dominant direction of the contour lines is the line $\lambda * \mu=$ constant. This line will keep the scaled primaries, $\lambda \mu \hat{\mathbf{X}}_{0} \hat{S}$, constant. For values of $\lambda>\mu$, the scaled multiples $\lambda \hat{\mathbf{X}}_{0} \mathbf{P}$ will deviate the contours.

Although the number of iterations in EPSI is higher (for the marine data with moderate water depth 180 iterations and for the shallow water marine data 240 iterations) than the number of iterations in SRME (for both data sets three iterations), we see reasons to assume that the calculation time does not have to be bigger by a factor of 60-80. First, because $\hat{\mathbf{X}}_{0}$ is spiky in the time domain, its convolution with the total data can be carried out in the time domain, saving on the costs of going through a Fourier transform. Second, the correlation $\mathbf{P} \mathbf{P}^{H}$ has to be calculated only once. Furthermore, the number of iterations can be decreased by making the number of events that are selected from $\Delta \mathbf{X}_{0}$ a function of the iteration number. Also note that it takes a lot of iterations to build the direct primary estimation; however, the conservative primary estimation takes its form much faster because most of the multiples are the result of a few dominant primaries that are estimated in the beginning of the process.

EPSI is a very open method in the sense that it is easy to build in other applications, such as the reconstruction of missing near offsets, or to apply it to other forms of data, such as blended (van Groenestijn and Verschuur, 2009c) or passive data (van Groenestijn and Verschuur, 2009d). Therefore, we think that EPSI can also be extended to the full 3D marine case in which the missing data between the streamer lines might be treated in a similar way as the missing near offsets in the 2D method.

Furthermore, we see possibilities to incorporate source-directivity effects and direct wave estimation for the shallow-water case. Instead of selecting and summing the elements of the main diagonal of $\Delta \hat{\mathbf{S}}$ in equation 10 , the main diagonal and its neighboring diagonals can be selected to capture the source directivity. If the acquisition is done in such a way that an upgoing and a downgoing wavefield can

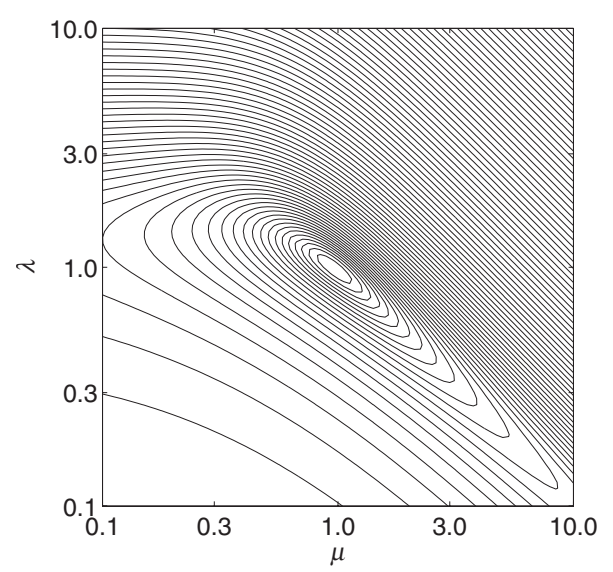

Figure 12. Logarithmic contour plot of the objective function as function of the final estimated primary impulse responses, $\hat{\mathbf{X}}_{0}$, and the final estimated source wavelet, $\hat{S} ; J(\lambda, \mu)=\Sigma_{\omega} \Sigma_{j, k} \mid \mathbf{P}-\lambda \mu \hat{\mathbf{X}}_{0} \hat{S}$ $+\left.\lambda \hat{\mathbf{X}}_{0} \mathbf{P}\right|_{j, k} ^{2}$. The parameter $\lambda$ varies $\hat{\mathbf{X}}_{0}$, and the parameter $\mu$ varies $\hat{S}$. The contour plot has a minimum in point, $\mu=1, \lambda=1$. Away from this point, each contour line represents an increase of $0.7 \mathrm{~dB}$. be obtained and the direct wave is only present in the downgoing wavefield, then EPSI can be rewritten as a division of the upgoing by the downgoing data to include the direct wave in its formulations (van Groenestijn and Verschuur, 2009b). This direct wave will capture the source directivity very nicely.

\section{CONCLUSIONS}

We have discussed the theory and algorithm behind EPSI and its application to the reconstruction of missing near offsets on a detailed level. We have illustrated the theory with two marine data sets. The results obtained with EPSI and iterative SRME for marine data with moderate water depth were similar in quality. An interesting property of EPSI is the residual, which can be used as a quality-control tool to see if certain events are not properly explained, indicating an inconsistency in the data.

For the shallow-marine data, EPSI showed a much better result than iterative SRME. This is mainly because the reconstruction of missing near offsets can be incorporated into EPSI so that they explain the multiples observed in the data.

\section{ACKNOWLEDGMENTS}

The authors thank the sponsors of the DELPHI consortium for the support of this research and for the stimulating discussions at the consortium meetings. Shell International Exploration and Production in Rijswijk, Netherlands, is acknowledged for arranging and giving permission to publish the marine data sets. The first author also wishes to acknowledge Shell for the processing facilities, feedback, and hospitality that were provided during an internship. Anatoly Baumstein, Roald van Borselen, Kris Innanen, and the anonymous reviewer are thanked for their suggestions that have improved the paper.

\section{APPENDIX A \\ PREPROCESSING OF FIELD DATA AND IMPLEMENTATION DETAILS}

To apply a 2D algorithm to a 3D data set, some preprocessing was done on both field data sets described in this paper. Here follows a list of the preprocessing steps that were taken:

- removal of random noise

- deconvolution for the source bubble effects

- removal of the direct wave by a muting operation

- differential NMO to bring the receivers and sources on the same grid because each line was selected from a 3D survey

- $f$ - $k$ interpolation of missing shots in the common-offset domain. The moderately deepwater marine data set has a shot spacing of $50 \mathrm{~m}$ and a receiver spacing of $12.5 \mathrm{~m}$. Therefore, three extra shots per shot needed to be interpolated. The shallow-water marine data set has a shot spacing of $37.5 \mathrm{~m}$ and a receiver spacing of $12.5 \mathrm{~m}$ so that two extra shots per shot were interpolated.

- parabolic Radon domain-based interpolation of missing near offsets (Kabir and Verschuur, 1995). For the shallow-marine data set, the first $0.4 \mathrm{~s}$ were set to zero again because the interpolated near offsets are not reliable there; they were part of the data to be estimated by the EPSI process. The same data set, but without the first $0.4 \mathrm{~s}$ set to zero, was used as input for iterative SRME. 
- A factor $\sqrt{t}$ was applied to the data as a 3D to $2 \mathrm{D}$ amplitude correction.

- Reciprocity was used to convert the data from a marine end-on geometry to a split-spread geometry.

Because the current implementation of the EPSI algorithm is based on matrices, the data had to be fed to EPSI in blocks. The maximum offset present in these blocks $(148 \times 12.5 \mathrm{~m}=1850 \mathrm{~m}$ for both field data sets) was also used as the maximum offset fed into iterative SRME. The blocks overlapped in such a way that a primary estimate was present for all the offsets between $0 \mathrm{~m}$ and $49 \times$ $12.5 \mathrm{~m}=612.5 \mathrm{~m}$. For a proper comparison of both the EPSI and iterative SRME results, only these offsets were stacked.

Iterative SRME (Verschuur and Berkhout, 1997) was used in three iterations using a wavelet of 31 samples for the global matching and a filter of three samples for the local matching in windows of 250 samples by 25 traces.

\section{REFERENCES}

Amundsen, L., 2001, Elimination of free-surface related multiples without need of the source wavelet: Geophysics, 66, 327-341.

Berkhout, A. J., 1982, Seismic migration: Imaging of acoustic energy by wave field extrapolation: A: Theoretical aspects: Elsevier.

- 2006, Seismic processing in the inverse data space: Geophysics, 71 no. 4, A29-A33.

Berkhout, A. J., and D. J. Verschuur, 1997, Estimation of multiple scattering by iterative inversion: Part I - Theoretical considerations: Geophysics, 62, 1586-1595.

Berryhill, J. R., and Y. C. Kim, 1986, Deep-water peg legs and multiples: Emulation and suppression: Geophysics, 51, 2177-2184.

Biersteker, J., 2001, Magic: Shell's surface multiple attenuation technique: 71st Annual International Meeting, SEG, Expanded Abstracts, 13011304.

Curry, W., and G. Shan. 2008, Interpolation of near offsets using multiples and prediction-error filters: 78th Annual International Meeting, SEG, Expanded Abstracts, 2421-2425.
Guitton, A., and D. J. Verschuur, 2004, Adaptive subtraction of multiples using the $L_{1}$-norm: Geophysical Prospecting, 52, 27-38.

Kabir, M. M. N., and D. J. Verschuur, 1995, Restoration of missing offsets by parabolic radon transform: Geophysical Prospecting, 43, 347-368.

Lokshtanov, D., 1999, Multiple suppression by data-consistent deconvolution: The Leading Edge, 18, 115-119.

Nekut, A. G., and D. J. Verschuur, 1998, Minimum energy adaptive subtraction in surface-related multiple attenuation: 68th Annual International Meeting, SEG, Expanded Abstracts, 1507-1510.

Shan, G., and A. Guitton, 2004, Migration of surface-related multiples: tests on the SIGSBEE2B dataset: 74th Annual International Meeting, SEG, Expanded Abstracts, 1285-1288.

van Borselen, R. G., J. T. Fokkema, and P. M. van den Berg, 1996, Removal of surface-related wave phenomena - The marine case: Geophysics, 61, $202-210$.

van Groenestijn, G. J. A., and D. J. Verschuur, 2008, Towards a new approach for primary estimation: 78th Annual International Meeting, SEG, Expanded Abstracts, 2487-2491.

-, 2009a, Estimating primaries by sparse inversion and application to near-offset data reconstruction: Geophysics, 74, no. 3, A23-A28.

,$- 2009 \mathrm{~b}$, Estimation of primaries by sparse inversion applied to up/ down wavefields: 79th Annual International Meeting, SEG, Expanded Abstracts, 3143-3147.

-2009 c, Estimation of primaries by sparse inversion from blended data: 71st Conference and Exhibition, EAGE, Extended Abstracts, S008.

, 2009d, Estimation of primaries by sparse inversion from passive seismic data: 79th Annual International Meeting, SEG, Expanded Abstracts 1597-1601.

Verschuur, D. J., 1991, Surface-related multiple elimination: An inversion approach: Ph. D. thesis, Delft University of Technology.

Verschuur, D. J., 1992, Surface-related multiple elimination in terms of Huygens' sources: Journal of Seismic Exploration, 1, 49-59.

- 2006, Seismic multiple removal techniques — Past, present and future: EAGE Publications BV.

Verschuur, D. J., and A. J. Berkhout, 1997, Estimation of multiple scattering by iterative inversion: Part II - Practical aspects and examples: Geophysics, 62, 1596-1611.

Verschuur, D. J., A. J. Berkhout, and C. P. A. Wapenaar, 1992, Adaptive surface-related multiple elimination: Geophysics, 57, 1166-1177.

Weglein, A. B., F. A. Gasparotto, P. M. Carvalho, and R. H. Stolt, 1997, An inverse scattering series method for attenuating multiples in seismic reflection data: Geophysics, 62, 1975-1989.

Wiggins, J. W., 1988, Attenuation of complex water-bottom multiples by wave-equation-based prediction and subtraction: Geophysics, 53, 15271539 